\title{
Pathology of Avipoxvirus Isolates in Chicken Embryonated Eggs
}

\author{
Bhavesh Sharma $^{1}$, Nawab Nashiruddullah ${ }^{1 *}$, Jafrin Ara Ahmed ${ }^{2}$, \\ Sankalp Sharma ${ }^{1}$ and D. Basheer Ahamad ${ }^{3}$
}

\author{
${ }^{1}$ Division of Veterinary Pathology, ${ }^{2}$ Division of Veterinary Physiology \& Biochemistry, \\ Faculty of Veterinary Sciences and Animal Husbandry, Sher-e-Kashmir University of \\ Agricultural Science and Technology of Jammu, RS Pura-181102, India \\ ${ }^{3}$ Department of Veterinary Pathology, \\ Veterinary College and Research Institute, Tamil Nadu University of Veterinary and Animal \\ Sciences, Tirunelveli, India
}

*Corresponding author

\section{A B S T R A C T}

\section{Keywords}

Avipoxvirus,

Chicken

embryonated eggs

(CEE),

Chorioallantoic membrane (CAM),

Cytopathic effect

(CPE),

Fowlpoxvirus

(FWPV),

Pigeonpoxvirus (PGPV)

\section{Article Info}

Accepted:

15 August 2019

Available Online:

10 September 2019
Fowlpox virus (FWPV) and Pigeonpox virus (PGPV) isolates from domesticated fowls and pigeons from Jammu region were inoculated on chorioallantoic membrane (CAM) of chicken embryonated eggs (CEE) through three passage levels to observe their cytopathic effects (CPE) and underlying pathology. Development of PGPV induced lesions was earlier than FWPV, and more severe. PGPV progressed from small white opaque lesions from first passage; to CAM thickening, haemorrhages and very prominent and extensive pock lesions at second; and by third passage, focal areas of necrosis was evident. With FWPV, opaque thickening with haemorrhages at first passage; to tiny, discrete, white foci on second; and at third passage, small, round, raised circular white pock were distinctly visible. CAM histology revealed PGPV induced hyperplasia of the chorionic epithelium; oedema, thrombosis, massive fibroblastic proliferation, necrosis and islands of vacuolated epithelial cells containing eosinophilic intra-cytoplasmic inclusions in the mesoderm. FWPV induced lesions were similar but necrosis of the epithelial layers was more widespread; vascular lesions in the mesoderm were more profound, proliferation of fibrous tissue was less, and the absence of inclusion bodies. It was observed that pock morphology on CAM may not be a reliable basis of Avipoxvirus species differentiation, although such variations both grossly and histologically exist.

\section{Introduction}

Avian pox is a group of DNA viruses of the genus Avipoxvirus, under family Poxviridae, and subfamily Chordopoxvirinae, and has long been observed in several avian species.
They have been observed in more than 230 of the known 9000 species of birds, spanning 23 orders (van Riper and Forrester, 2007), yet little is known about the genome diversity, host range and host specificity of the causative agents. It is characterized by the formation of 
proliferative lesions, scabs on the skin, and diphtheritic lesions in the upper digestive and respiratory tracts. The genus currently comprises of ten recognized species (Smith et al., 2008), of which Fowlpox virus (FWPV) is the type species. Antigenic relationship exists among Avipoxvirus, and some avian poxviruses are more closely related to some than others. There is evidence of considerable heterogeneity among species of avian poxviruses.

Avipoxvirus replicates in the cytoplasm of infected cells and depending on the isolateproduces typical cytopathic effect (CPE) 4 to 6 days post infection. The virus can be grown on the chorioallantoic membrane (CAM) of embryonated chicken egg and in a variety of cell culture models. Pox virus culture on chorioallantoic membrane (CAM) of chicken embryonated eggs (CEE) is one of the identification tools of choice (Woodruff and Goodpasture, 1931; Cunningham 1973). Since the chicken embryo at 10-15 days old lacks a functional specific immune system (Eerola $e t$ al., 1987; Dibner et al., 1998) it provides an opportunity to analyze virus-induced host responses in the absence of specific adaptive immune responses (Fredrickson et al., 1992). Thickening of the infected CAM produces typical focal and diffuse lesions. These lesions are fairly diagnostic and can be readily confirmed by histological observations or electron microscopy (OIE, 2008).

In our laboratory, domesticated fowls, pigeons and turkey birds were screened for avipoxvirus infection from different areas in Jammu region, India, and on the basis of the phylogenetic analysis of the partial $P 4 b$ gene sequences, three different isolates were identified as Fowlpox virus (FWPV), Pigeonpox virus (PGPV) and Turkeypox virus (TKPV) respectively (Sharma et al., 2019); and it was also found that the FWPV and TKPV were phylogenetically 99\% related within clade 1, while PGPV was found to belong to clade 2. Since differences in CPE on CAM by different Avipoxviruses (APV) have been earlier reported (Manarolla et al., 2010; Abdallah and Hassanin, 2013; Offerman et al., 2013) it was proposed to attempt isolation of the viruses on CEE. Whereas the TKPV samples comprised of formalin preserved and archival specimens, growth of only the FWPV and PGPV isolates were tried in the present study to observe their differential pathology, if any.

\section{Materials and Methods}

\section{Suspected clinical cases}

Suspected samples of Avipoxvirus infection were collected from backyard poultry (fowl) and pigeons from Rajouri and Jammu districts. Samples were collected at different times from April 2016 to May 2017. Fowlpox (FP) outbreaks were covered from six select areas of which five were from Rajouri district and one from Jammu district. Similarly, samples of Pigeonpox (PP) were taken from four select places in Ranbir Singh Pura in Jammu which was very near to the Indo-Pak International border. The open-ranged pigeons although domesticated, sometimes crossed over to each side of the border.

\section{Collection of clinical samples}

Clinical materials included scabs, skin lesions, or occasional incisional biopsies, and lesions from oral membranes from Avipox suspected live or dead birds.

\section{Isolation of putative APV (FWPV and PGPV) from clinical samples}

Clinical scab/skin samples of affected birds were processed for virus isolation. A 10-20\% solution of the scab samples in PBS was prepared by mechanical homogenization using a sterilized mortar pestle. Homogenized samples were centrifuged at $3,000 \times \mathrm{g}$ for 15 
min. The supernatant was then passed through a $0.45 \mu \mathrm{m}$ syringe filter (Millipore) and incubated with streptomycin, penicillin antibiotic solution (@ 200 IU of penicillin and $200 \mu \mathrm{g}$ of streptomycin per $\mathrm{ml}$ suspension) for $1 \mathrm{hr}$ at $37^{\circ} \mathrm{C}$. Processed samples were used for infecting CAM for virus isolation.

\section{Chorio-allantoic membrane inoculation of} virus in chicken embryonated eggs

For virus isolation in CAM, the protocol described in Cunningham (1973) by the artificial air sac route was employed. Approximately 10-12 day old chicken embryonated eggs from Government Poultry Hatchery Unit, Belicharana, under Animal Husbandry Department, Jammu were procured for virus inoculation. The eggs were acquired after ensuring that there was no history of Fowlpox infection in the parent flock. Out of various field samples, one each of FWPV and PGPV were adopted (in triplicate) on CAM through three passage levels. The isolates have been earlier verified by partial amplification of $P 4 b$ gene and confirmed as FWPV and PGPV by alignment with published sequences (Sharma et al., 2019). The eggs were candled daily and opened on 5 d.p.i. Cutaneous lesions were processed and used as inoculum for first passage. Blind passages or CAM showing confluent growth of pocks was harvested as a source of Avipoxvirus for next passage. The development of characteristic lesions if any was noted for each passage level. For each Avipox inoculation and at every passage level, a negative control CEE was incubated alongside, with injection of sterile PBS on the CAM.

\section{Results and Discussion}

\section{Chicken embryonated egg inoculation}

The lesions on CAM inoculated with both fowl (FWPV) and pigeon (PGPV) isolates at each of the three passage levels are depicted in Figure 1.

\section{FWPV}

On the first and second passage, the CAM appeared opaque, thick oedematous and haemorrhagic (Fig. 1A, B). Tiny, discrete, white foci were observed on CAM that was not always discernible (Fig. 1C, D). During third passage, small, round, raised circular white lesions or 'pocks', of varying morphology were distinctly visible (Fig. 1E, F). Size of lesions was approximately 1-3 mm in diameter. No CPE was observed in control CEE incubated at each passage level.

\section{PGPV}

On first passage, in some instances the CAM showed small white opaque lesions (Fig. 2A) or appeared opaque, thick, oedematous and often with petechial haemorrhagic (Fig. 2B). Subsequent passage revealed very prominent and extensive pock lesions (Fig. 2C). In one instance a substantial thickening and diffuse opaqueness of the CAM was observed, completely obscuring any details (Fig. 2D). By $3^{\text {rd }}$ passage, they were characterized by focal areas of necrosis and opacity, thickening and oedema (Fig. 2E, F). Size of pock lesions was nearly 1-5 mm in diameter. Development of lesions was earlier and more severe in Pigeonpox samples than those of Fowlpox. No CPE was observed in control CEE incubated at each passage level.

\section{CAM histopathological lesion}

\section{PGPV}

The Pigeonpox lesion in the chorio-allantoic membrane is noticeable with hyperplasia of the epithelial cells of the ectoderm (chorionic epithelium) over the inoculated area (Fig. 3A). Sometimes increased in CAM thickness is 
observed manifold than the normal. The epithelial cells appear hypertrophied. The mesoderm is oedematous and blood vessels appear thrombosed (Fig. 3B). There is necrosis of the ectoderm over the inoculation site with characteristically a massive proliferation of fibroblasts in the mesoderm (Fig. 3C).

Figure.1 Chorio-Allantoic Membrane (CAM) inoculation of Fowlpox scab samples in 1012 day old embryonated chicken eggs

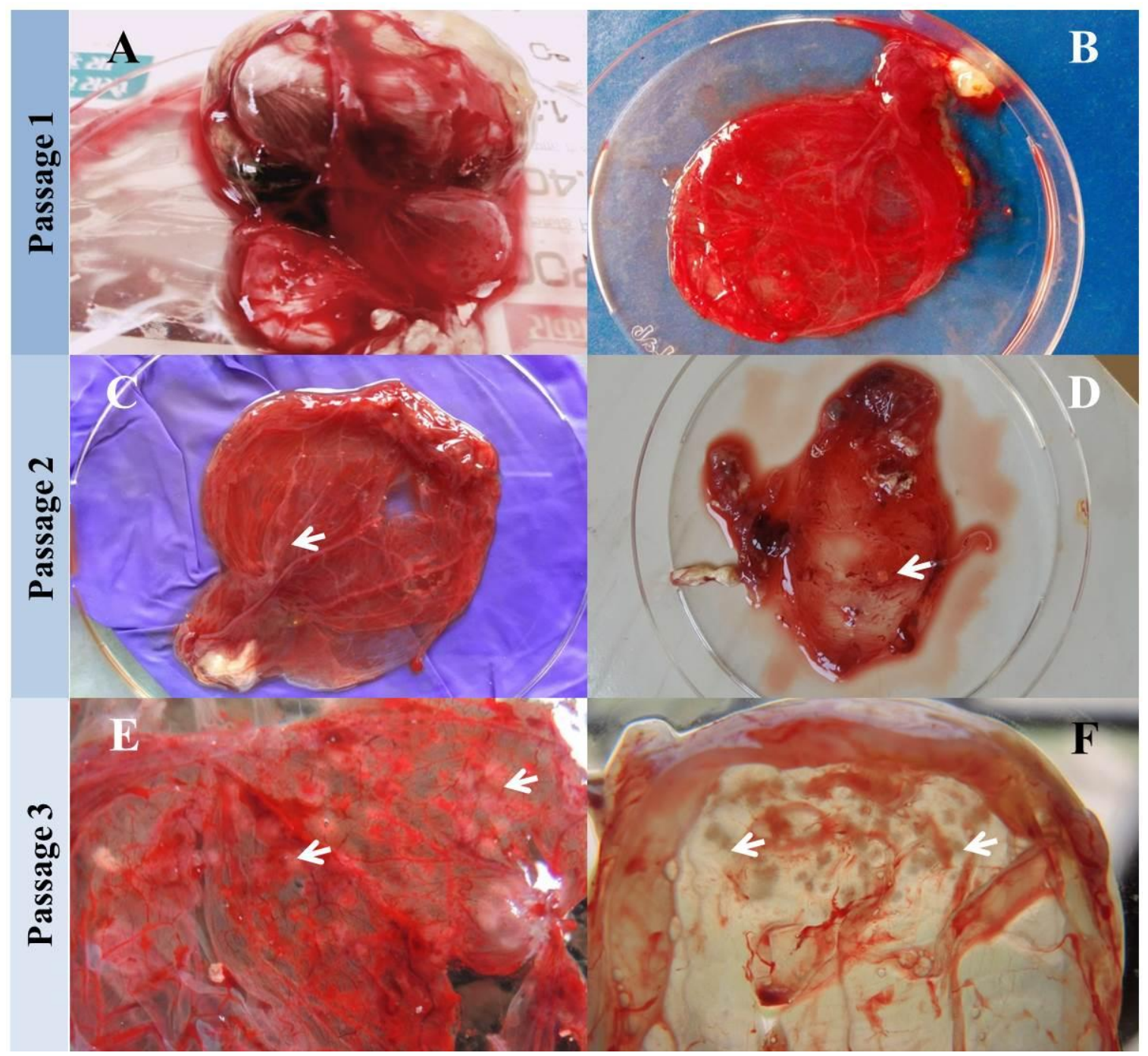

Passage 1 (A, B) oedematous and haemorrhagic CAM

Passage 2 (C, D) small white necrotic foci (white arrows) on thickened CAM Passage 3 (E, F) small round and multiple white opaque spots (white arrows) on CAM 
Figure.2 Chorio-Allantoic Membrane (CAM) inoculation of Pigeonpox scab samples in 10-12 day old embryonated chicken eggs

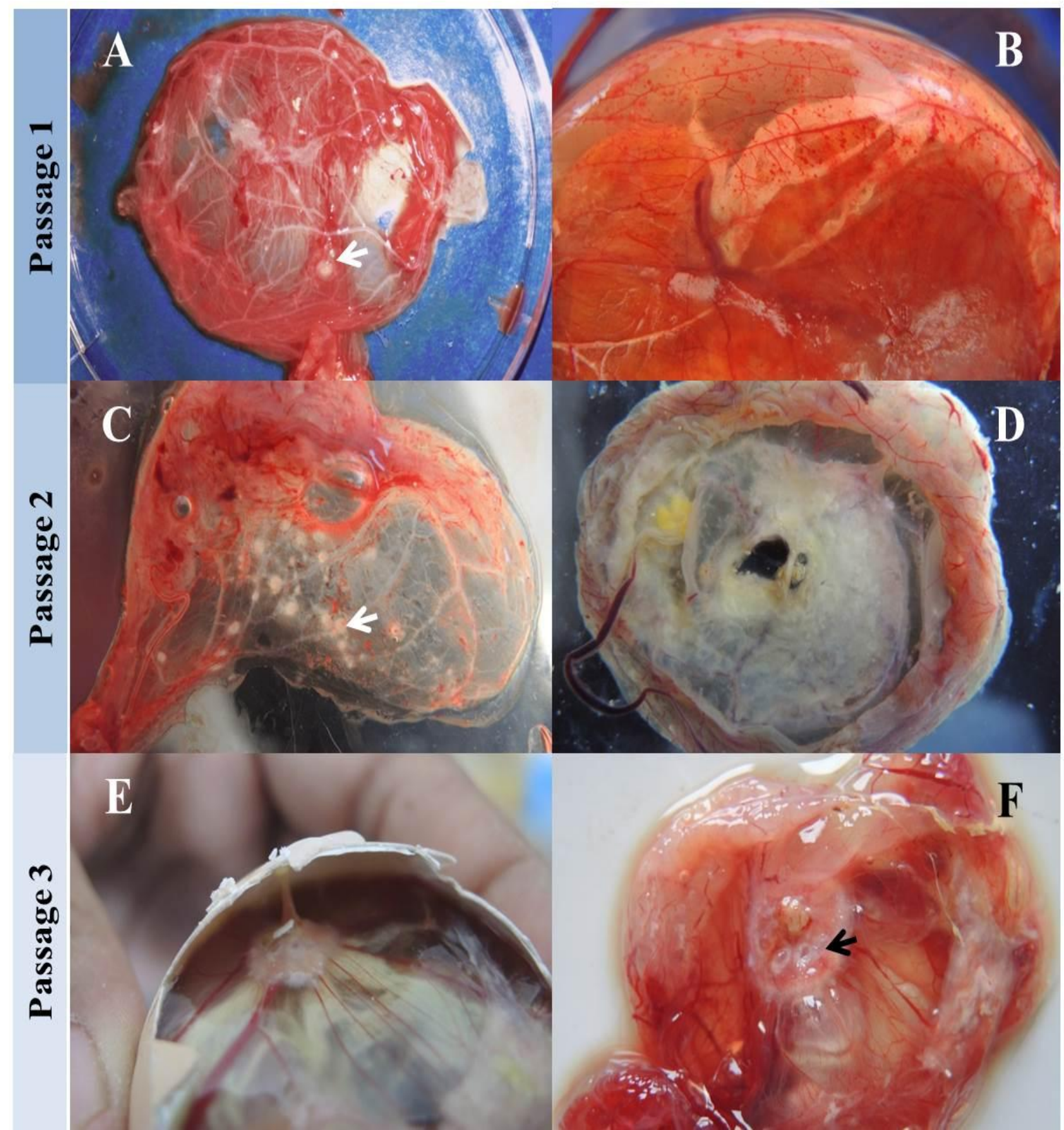

Passage 1 (A) Thickened CAM with white necrotic foci (B) oedematous and haemorrhagic CAM

Passage 2 (C) Small white necrotic foci (pocks) on CAM; (D) Diffusely thickened and opaque CAM with obscured pock lesions

Passage 3 (E, F) Focal thickening of CAM with large, ulcerative pock lesions 
Figure.3 Histopathological changes in the Chorio-allantoic membrane of 10-12 day old embryonated chicken egg infected with Pigeon poxvirus (PGPV) and Fowl poxvirus (FWPV) inoculum
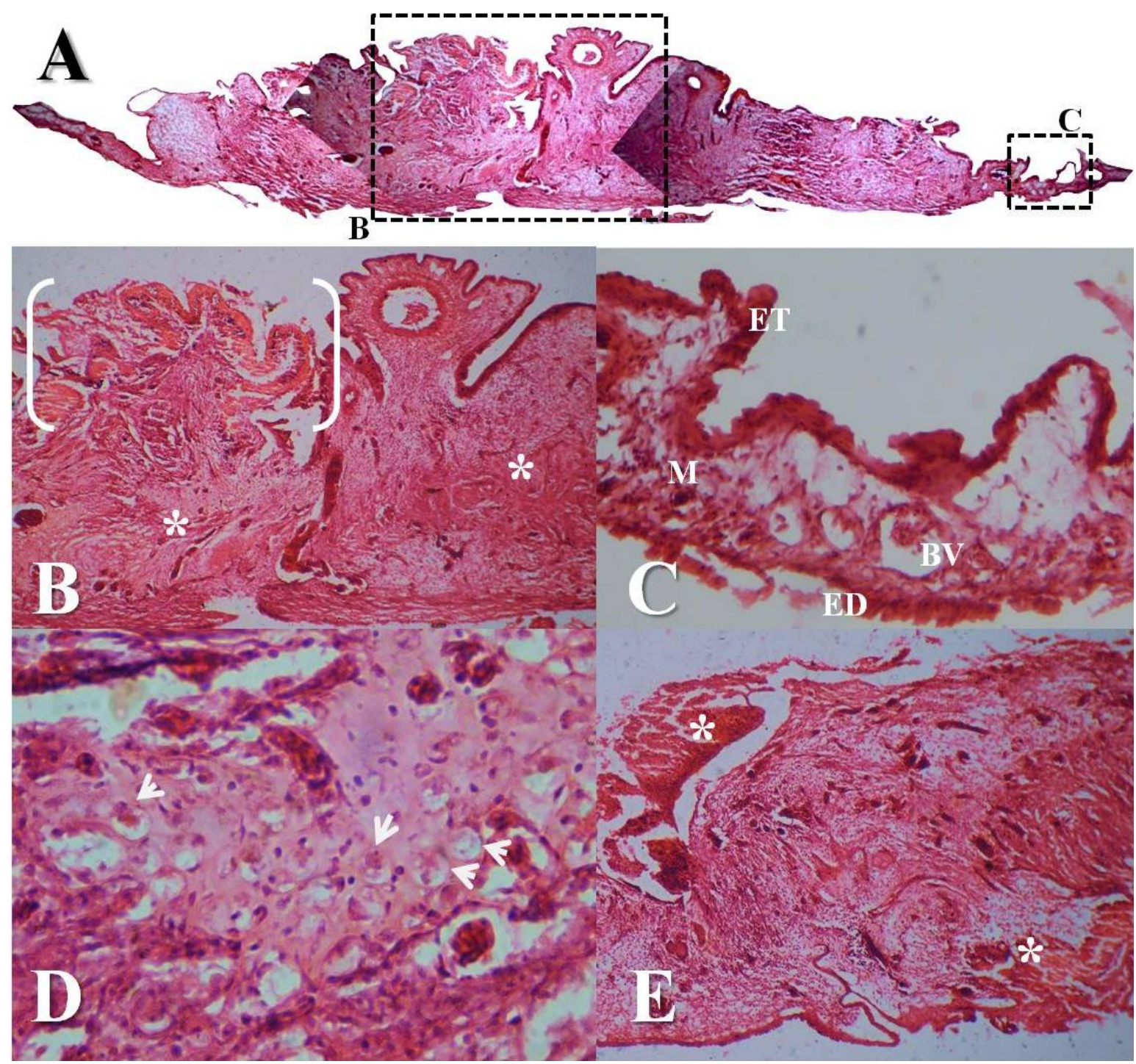

(A) Composite picture of a PGPV inoculated CAM showing its thickening over the inoculation site. Note the near normal thickness over site marked 'B'. H\&E, 40X; (B) Magnified image of 'B' showing oedema and thrombosis of blood vessels (BV) in the mesoderm (M) and layers of the ectoderm (ET) and entoderm (ED). $\mathrm{H} \& \mathrm{E}, 100 \mathrm{X}$; (C) Magnified image at 'C' showing area of necrosis of ectodermal chorionic epithelium (white bracket) over inoculation site, and proliferation of fibrous tissue $\left(^{*}\right)$ in the mesoderm (M). H\&E, 100X. (D) PGPV inoculated CAM showing eosinophilic, intra-cytoplasmic inclusions (white arrow) in epithelial cells forming islands within the mesoderm. Note infiltration of mononuclear cells in the matrix. H\&E, 400X; (E) FWPV inoculated CAM showing massive haemorrhage (*) in mesoderm with sloughing of both entoderm and ectodermal layers. H\&E, 100X.

Moderate mononuclear leukocytes are seen in the mesoderm interspersed between the fibrocellular matrix. In certain sections massive haemorrhage with sloughing of both the chorionic epithelium and the entoderm (allantoic epithelium) is seen. No inclusion 
bodies could be found in the epithelial cells of these layers. Islands of epithelial cells as nests were seen in the mesoderm also surrounded by connective tissue elements. The cells were swollen with prominent vacuolation and often containing many eosinophilic intracytoplasmic inclusion typical of pox virus (Fig. 3D).

\section{FWPV}

The CAM lesions inoculated with Fowlpox appear to be similar to those inoculated with Pigeonpox. However, necrosis of the epithelial layers was more widespread. Vascular lesions in the mesoderm were more profound including oedema, thrombosis and haemorrhages (Fig. 3E). Proliferation of fibrous tissue and mononuclear leucocytes was less.

In the present study, there was an attempt to differentiate the two virus isolates originating from fowl and pigeons, based on their CAM pathology. The Pigeonpox isolate seemingly caused an earlier and more pronounced CPE at the first passage level, although in a heterologous host (chicken egg). In contrast, the Fowlpox isolate had a delayed CPE response (by 2-3 passage level). Besides, one Pigeonpox isolate at second passage caused substantial thickening and diffuse opaqueness so as to obscure any underlying lesions on the CAM, if present. Such opaque lesions were similarly observed by Offerman et al., (2013), who had to dilute the virus titre to reduce the membrane thickening and discern the distinct white pocks. In another of our observations, the Pigeonpox isolates at third passage caused focal opaque thickening of the CAM with characteristic ulcerative pocks. These lesions were very different for those described for other PGPVs. Based on the pock morphology, our PGPV (characterized with white pocks and very severe necrosis) is distinctly different to those reported from South African
PGPV isolates with white pocks and variable pathology (Offerman et al., 2013) or Egyptian PGPV isolates producing yellowish nodular pocks (Abdallah and Hassanin, 2013).

The reason for such observed variations is difficult to ascertain, but there could be various contributing factors. Plausible reasons could include dose and titre of infection (Offerman et al., 2013), acquired virulence due to integration of reticuloendotheliosis virus genome (Zhao et al., 2014), passage in species different from those in which they originated (Buddingh, 1938), different levels of immune response in the CAM tissue (Palumbo et al., 1989; Roth et al., 2012), or caused by the genetic make-up of the virus (Offerman et al., 2013). Available literatures even suggest the significance of pock colour and differential thickening of CAM as a basis of virus variants, at least in orthopoxviruses (Archard and Mackett, 1979; Archard et al., 1984).

There have been many instances where differences in CPE on CAM by different Avipoxviruses have been reported (Goodpasture, 1959; Mayr, 1963; Cunningham, 1973; Manarolla et al., 2010; Abdallah and Hassanin, 2013; Offerman et $a l ., 2013)$. It is worthwhile to mention that the differences in CPE reactions in such differential systems are either too subtle or very similar to allow clear-cut differentiations between Avipoxvirus species. Tripathy and Reed (2003) summarized that due to these ambiguities, there had been limited success on attempts to classify Avipoxvirus isolates on the basis of their lesions on the CAM of inoculated eggs, in avian cell cultures, or by subcutaneous versus intravenous inoculation of several susceptible bird species. On similar lines, Offerman et al., (2013) concluded no correlation between gross morphology, histopathology, pock morphology and phylogenetic grouping of avipoxviruses, nor 
was there any correlation between geographical distribution and virus phenotype or genotype.

The underlying proliferative, necrotic and vascular changes on the CAM of virus infected CEE, including thickening of the membrane and immune cell infiltration, are suggestive of an acute inflammatory response (Offerman et al., 2013). In the present study, the salient histopathological feature in the PGPV infected CAM was the severe proliferation of mesodermal connective tissue and the presence of inclusions in epithelial nests within the mesoderm. No inclusions however were noticed in the Fowlpox infected CAM and the inflammatory cellular response was also mild to moderate. Significant differences in Avipoxviruses on the basis of gross and histological changes in the CAM have been earlier reported (Offerman et al., 2013). The authors also remarked that viruses causing severe macroscopic proliferation of the CAM were noted to have extensive mesodermal hyperplasia and less epidermal hyperplasia. This fact is exactly substantiated in the present findings. Besides, Offerman et al., (2013) observed that FWPV does not produce extensive membrane thickening, but a degree of hyperplasia is observed when compared with uninfected CAM tissue. Further, they suggested that Avipoxviruses causing inflammation may contain additional growth factor-like genes responsible for the increased inflammation. Variations in degree of inflammatory response may be attributed to viral immunomodulatory mediators that modulate the host response and it is likely that their presence and expression in different virus strains may vary. Such investigations, would however need very exhaustive and detailed genomic and immunomodulator analysis.

In conclusion, the visible pock morphology on CAM may not be a reliable basis of
Avipoxvirus species differentiation, although such variations both grossly and histologically exist. However, the reason for such variation would be intriguing, requiring an in-depth analysis of both pathogen and host factors and their intricate interaction.

\section{Conflict of Interest}

The authors declare no conflict of interest

\section{References}

Abdallah, F.M. and Hassanin, O. 2013. Detection and molecular characterization of avipoxviruses isolated from different avian species in Egypt. Virus Genes, 46: 63-70.

Archard, L. C. and Mackett, M. 1979. Restriction endonuclease analysis of red cowpox virus and its white pock variant. Journal of General Virology. 45: 51-63.

Archard, L. C., Mackett, M., Barnes, D. E. and Dumbell, K. R. 1984. The genome structure of cowpox virus white pock variants. Journal of General Virology. 65: $875-886$.

Buddingh, G.J. 1938. A study of the behavior of fowl-pox virus modified by intracerebral passage. Journal of Experimental Medicine, 67: 933-940.

Cunningham, C. H. 1973. A Laboratory Guide in Virology.7th Edition. Burgess Publishing Co., Minneapolis, Minnesota.

Dibner, J.J., Knight, C.D., Kitchell, M.L., Atwell, C.A., Downs, A.C., Ivey, F.J. 1998. Early feeding and development of the immune system in neonatal poultry. Journal of Applied Poultry Research, 7: 425-436.

Eerola, E., Veromaa, T. and Toivanen, P. 1987. Special features in the structural organization of the avian lymphoid system. In Avian Immunology: Basis and Practice. Edited by Toivanen, A. \& Toivanen, P. Boca Raton, FL: CRC 
Press. Pp. 9-21.

Fredrickson, T. N., Sechler, J. M., Palumbo, G. J., Albert, J., Khairallah, L. H., and Buller, R. M. 1992. Acute inflammatory response to cowpox virus infection of the chorioallantoic membrane of the chick embryo. Virology, 187: 693-704.

Goodpasture, E. W. 1959. Cytoplasmic inclusions resembling Guarnier bodies and other phenomena induced by mutants of the virus of fowl pox. American Journal of Pathology, 35: 213- 223.

Manarolla, G., Pisoni, G., Sironi, G. and Rampin, T. 2010. Molecular biological characterization of avian poxvirus strains isolated from different avian species. Veterinary Microbiology, 140: 1-8.

Mayr, A. (1963). Neue Verfahreu Fuerdie Differenzierung der Geluegel Pox keviren. Berlin. Muench. Tieraerzil Wochschr, 76: 316-324.

Offerman, K., Carulei, O., Gous, T.A., Douglass, N. and Williamson, A.L. 2013. Phylogenetic and histological variation in avipox viruses isolated in South Africa. Journal of General Virology, 94: 2338-2351.

OIE Terrestrial Manual. 2008. Fowl pox. Chapter 2.3.10. Pp. 531-537.

Palumbo, G. J., Pickup, D. J., Fredrickson, T. N., McIntyre, L. J. and Buller, R. M. 1989. Inhibition of an inflammatory response is mediated by a $38-\mathrm{kDa}$ protein of cowpox virus. Virology, 172: 262-273

Roth, S. J., Klopfleisch, R., Osterrieder, N. and Tischer, B. K. 2012. Cowpox virus serpin CrmA is necessary but not sufficient for the red- poxk phenotype on chicken chorioallantoic membranes. Virus Research, 163: 254-261.

Sharma, B., Nashiruddullah, N., Bhat, M. A., Roychoudhury, P., Ahmed, J. A., Sood, S. and Mehmood, S. 2019. Occurrence and phylogenetic analysis of avipoxvirus isolated from birds around Jammu. Virus Disease, 30(2): 288-293.

Smith, G. L., Beard, P. and Skinner, M. A. 2008. Poxviruses. In: Encyclopedia of Virology, 3rd Edition. B. W. J. Mahy. And M.H.V. Van Regenmortel (Eds.), Academic Press. Pp. 322-330.

Tripathy, D.N. and Reed, W.M. (2003). Pox. In: Diseases of Poultry. 11th Edition (Editors Saif Y.M., Barnes H.J., Glisson J.R., Fadly A.M., McDougald L.R. and Swayne S.E.). Iowa State Press,Ames, Iowa. Pp. 253-269.

van Riper, C. III. and Forreste, D. 2007. Avian Pox. In: Infectious Diseases of Wild Birds. Eds. Thomas, N.J., Hunter, B.D. and Atkinson, C.T. Wiley-Blackwell Publishing. 131-176.

Woodruff, A. M. and Goodpasture, E. W. 1931. The susceptibility of the chorioallantoic membrane of chick embryo to infection with the fowlpox virus. American Journal of Pathology, 7: 209-222.

Zhao, K., W, He., S, Xie., Song, D., Lu, H., Pan, W., Zhou, P., Liu, W., Lu, R., Zhou, J. and Ga, F. 2014. Highly pathogenic fowl pox virus in cutaneously infected chickens, China. Emerging Infectious Diseases, 20(7): 1208-1210.

\section{How to cite this article:}

Bhavesh Sharma, Nawab Nashiruddullah, Jafrin Ara Ahmed, Sankalp Sharma and Basheer Ahamad, D. 2019. Pathology of Avipoxvirus Isolates in Chicken Embryonated Eggs. Int.J.Curr.Microbiol.App.Sci. 8(09): 422-430. doi: https://doi.org/10.20546/ijcmas.2019.809.051 